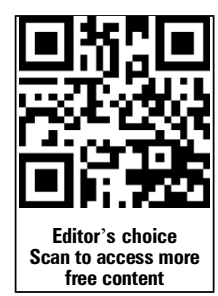

- Additional material is published online only. To view please visit the journal online (http://dx.doi.org/10.1136/ heartjnl-2015-308542).

${ }^{1}$ Basel Institute for Clinical Epidemiology and Biostatistics, University Hospital Basel, Basel, Switzerland

${ }^{2}$ Heart Care Western Australia, Perth, Australia ${ }^{3}$ Department of Clinical Research, University of Basel, Basel, Switzerland

${ }^{4}$ Department of Clinical Epidemiology and Biostatistics, McMaster University, Hamilton, Ontario, Canada

\section{Correspondence to} Dr Lars G Hemkens, Basel Institute for Clinical Epidemiology \& Biostatistics, University Hospital Basel, CH-4031 Basel, Switzerland; lars.hemkens@usb.ch

Received 17 August 2015 Revised 23 December 2015 Accepted 25 December 2015 Published Online First 1 February 2016

\section{SLinked}

- http://dx.doi.org/10.1136/ heartjnl-2015-309167

\section{CrossMark}

To cite: Hemkens $L G$ Ewald $\mathrm{H}$, Gloy VL, et al. Heart 2016;102:590-596.

\title{
Cardiovascular effects and safety of long-term colchicine treatment: Cochrane review and meta-analysis
}

\author{
Lars G Hemkens, ${ }^{1}$ Hannah Ewald, ${ }^{1}$ Viktoria L Gloy, ${ }^{1}$ Armon Arpagaus, ${ }^{1}$ \\ Kelechi K Olu, ${ }^{1}$ Mark Nidorf, ${ }^{2}$ Dominik Glinz, ${ }^{1}$ Alain J Nordmann, ${ }^{1}$ Matthias Briel ${ }^{1,3,4}$
}

\section{ABSTRACT}

Colchicine is an old anti-inflammatory drug that has shown substantial cardiovascular benefits in recent trials. We systematically reviewed cardiovascular benefits and harms of colchicine in any population and specifically in patients with high cardiovascular risk. We evaluated randomised controlled trials comparing colchicine over at least 6 months versus any control in any adult population. Primary outcomes were all-cause mortality, myocardial infarction and adverse events. Cardiovascular mortality was a secondary outcome. We included 39 trials with 4992 patients. The quality of evidence for mortality outcomes and myocardial infarction was moderate but lower for adverse events. Colchicine had no effect on all-cause mortality (RR 0.94, 95\% Cl 0.82 to $1.09 ; I^{2}=27 \% ; 30$ trials). Cardiovascular mortality was reduced in some but not all meta-analytical models (random-effects RR 0.34, 0.09 to $1.21,1^{2}=9 \%$; Peto's OR 0.24, 0.09 to $0.64, I^{2}=15 \%$; Mantel-Haenszel fixedeffect RR $0.20,0.06$ to $0.68, I^{2}=0 \% ; 7$ trials). The risk for myocardial infarction was reduced (RR 0.20, 0.07 to $0.57 ; 2$ trials). There was no effect on total adverse events (RR 1.52, 0.93 to $2.46, \mathrm{I}^{2}=45 \% ; 11$ trials) but gastrointestinal intolerance was increased (RR 1.83, 1.03 to $3.26, I^{2}=74 \%$; 11 trials). Reporting of serious adverse events was inconsistent; no event occurred over 824 patient-years (4 trials). Effects in high cardiovascular risk populations were similar (4 trials; 1230 patients). We found no evidence supporting colchicine doses above $1 \mathrm{mg} /$ day. Colchicine may have substantial cardiovascular benefits; however, there is sufficient uncertainty about its benefit and harm to indicate the need for large-scale trials to further evaluate this inexpensive, promising treatment in cardiovascular disease.

\section{INTRODUCTION}

Inflammation is a central mechanism of cardiovascular disease. ${ }^{1-3}$ Colchicine is an old drug with a range of anti-inflammatory effects. It is inexpensive and established for treating diverse conditions. ${ }^{4} 5$ For centuries, gout has been treated with autumn crocus extracts (Colchicum autumnale). ${ }^{5} \quad 6$ Colchicine has been used as long-term therapy, often in relatively high doses, for various chronic inflammatory conditions including familial Mediterranean fever (FMF), Behçet's disease, primary biliary cirrhosis or pericarditis. ${ }^{5-7}$

Colchicine for cardiovascular disease was first evaluated in 1992 in a small trial to prevent restenosis after elective angioplasty. ${ }^{8}$ The findings were interpreted as evidence of absence of cardiovascular benefits. $^{8}$ In 2013, however, two randomised clinical trials (RCTs) suggested that there may be substantial benefits of continuous low-dose colchicine treatment in the treatment of patients with stable coronary disease ${ }^{9}$ and in patients with diabetes undergoing angioplasty. ${ }^{10}$

There is no systematic review and meta-analysis assessing and synthesising the entire clinical trial experience on cardiovascular benefits of colchicine therapy. Although one recent systematic review examined its effects in cardiovascular disease, it was limited to trials in patients with known cardiac disease. ${ }^{\mathrm{w0}}$ Safety analyses in previous reviews on other specific indications of colchicine were also restricted to specific populations and did not consider the entire randomised evidence on adverse effects. However, many side effects (such as gastrointestinal intolerance) are probably unrelated to the underlying condition, and potential effects on arteriosclerosis are also not necessarily related to the indication.

We systematically reviewed and synthesised all available RCTs on long-term colchicine therapy. We assessed cardiovascular effects and safety in any population and, in addition, evaluated the benefits and harms of colchicine treatment specifically in patients with increased cardiovascular risk.

\section{METHODS}

This work was planned and conducted as a Cochrane review. ${ }^{11}$ We followed a detailed protocol published upfront ${ }^{12}$ and registered with PROSPERO (international database of prospectively registered systematic reviews). ${ }^{13}$ Further details on the methods are presented in the full Cochrane review. ${ }^{11}$

\section{Eligibility criteria}

We included RCTs and pseudorandomised trials in any adult population that compared continuous colchicine treatment over at least 6 months at any dose or way of application with placebo, no treatment, or any other treatment apart from colchicine. Patients at high risk of cardiovascular events (secondary prevention of cardiovascular disease events, established coronary heart disease) were a prespecified subgroup.

\section{Search strategy}

We searched for eligible studies in (1) MEDLINE, EMBASE, CENTRAL (no language restrictions; last search 30 January 2015; online supplementary file 1); (2) citations of key papers; ${ }^{8-10}$ (3) references of all included articles; (4) trial registries (ClinicalTrials. 
gov, WHO International Clinical Trials Registry; last search 22 January 2015).

We contacted the investigators of 29 studies where we could identify contact details. We asked for information on unreported outcomes, missing outcome data and unpublished studies. We sent them our extractions and bias assessments for review. Authors of 13 studies responded.

Teams of two independent reviewers screened titles and abstracts and evaluated full texts of potentially eligible articles. Discrepancies were resolved by consensus or with a third reviewer. One reviewer screened citations and references.

\section{Patient outcomes}

Primary outcomes were all-cause mortality, myocardial infarction and adverse events (any type, gastrointestinal, serious). Secondary outcomes were cardiovascular mortality, stroke, heart failure, non-scheduled hospitalisations (all-cause, cardiovascular) and non-scheduled cardiovascular interventions (ie, percutaneous coronary intervention (PCI))/angioplasty or coronary artery bypass graft). Composite outcomes were not considered. We accepted any definition of myocardial infarction, stroke or heart failure and separately assessed total, fatal and non-fatal events.

\section{Data extraction and assessment of bias and quality}

We extracted information on methods, participants, interventions, outcomes and funding. Two independent reviewers extracted outcome data and assessed the risk of bias. ${ }^{14} \mathrm{We}$ assessed publication bias using funnel plots (for outcomes with at least 10 studies). One reviewer extracted study characteristics and verified all extracted data, data in publication tables and bias assessments. Two reviewers used GRADE (Grading of Recommendations Assessment, Development and Evaluation) to assess the quality of evidence. ${ }^{15}$ Four studies not published in English were evaluated and three of them deemed ineligible by external native-speaking reviewers experienced in trial methodology. Disagreements were resolved by consensus.

\section{Statistical analysis}

We pooled treatment effects using random-effects models (DerSimonian and Laird; continuity correction of 0.5$)^{14}$ and calculated relative risks (RRs) with $95 \%$ CIs. ${ }^{16}$ For outcomes with event rates below $1 \%$ across all study groups, we applied Peto's approach. ${ }^{14}$ We preferred intention-to-treat analyses involving all randomised patients. Between-study heterogeneity was measured with $\mathrm{I}^{2}{ }^{17}$

We conducted subgroup analyses of trials with patients at high cardiovascular risk and of colchicine dose $(\leq 1 \mathrm{mg} /$ day vs $>1 \mathrm{mg} /$ day). We planned several sensitivity analyses (when there were at least three trials to be combined). Since reliability of meta-analytical methods may be reduced with sparse data we used Peto's approach and the Mantel-Haenszel method without zero correction ${ }^{14}$ for outcomes with event rates between $1 \%$ and $5 \% .{ }^{18}$ We evaluated effect modifications by type of control (active vs placebo or other), risk for selection bias (adequate randomisation sequence generation and allocation concealment vs other), double-blinding, blinded outcome assessment, attrition bias (incomplete vs complete outcome reporting) and type of publication (full journal publication vs abstract only). We used Stata V.13.1 (Stata Corp, College Station, Texas, USA) and Review Manager 5.3. $\mathrm{p}$ Values are two-tailed and $\mathrm{p}<0.05$ indicates statistical significance.

There were no relevant protocol deviations. All analyses were prespecified with the exception of sensitivity analyses using alternative meta-analytical models which we deemed useful when we observed event rates close to $1 \%$. In addition, we analysed the risk of bias in more detail.

\section{RESULTS}

We identified 2294 potentially relevant records in our literature searches. In study registries, we identified five ongoing trials that met our inclusion criteria; three of those specifically address cardiovascular disease.

We included 39 RCTs with 4992 patients (figure 1). Investigators provided unpublished outcome information for three trials, ${ }^{9} 1019$ two of them in patients with cardiovascular disease. ${ }^{9}{ }^{10}$ Most trials were small with a median of 84 patients ( IQR 54-129) and published before 2000 (table 1, online supplementary file 2).

Four RCTs including 1230 patients compared colchicine with placebo or usual care in a cardiovascular setting, ${ }^{8-10} 20$ that is, after elective angioplasty, ${ }^{8}$ in patients with diabetes undergoing bare metal stent implantation, ${ }^{10}$ in patients with stable chronic heart failure (71\% ischaemic) ${ }^{20}$ and patients with stable coronary disease. ${ }^{9}$ Most other studies $(n=25)$ used colchicine for hepatobiliary (mainly cirrhotic) diseases.

Colchicine doses were $\leq 1 \mathrm{mg} /$ day in 27 trials (69\%), and most of the other studies used $1.2 \mathrm{mg} /$ day. No study evaluated any of the cardiovascular end points in this review as primary outcome.

\section{Risk of bias assessment}

We found no indication for relevant selection, detection or performance bias (online supplementary file 3). Twenty-seven studies were double-blinded, 25 used placebo controls, 8 had blinded outcome assessment. The risk for bias due to incomplete outcome data was frequently high, because results were missing for substantial proportions of randomised patients or incompleteness was unbalanced between study groups. We

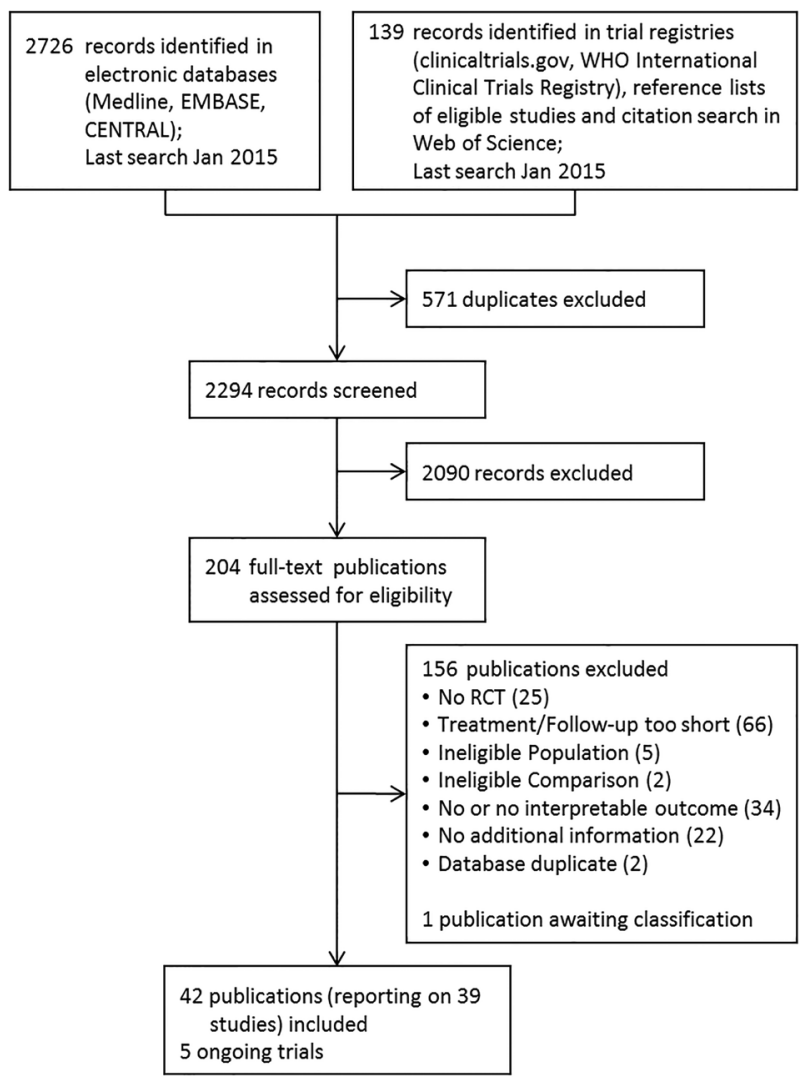

Figure 1 Study flow. RCT, randomised clinical trial. 
Table 1 Summary characteristics of the included studies

\begin{tabular}{|c|c|c|}
\hline & All studies & $\begin{array}{l}\text { Studies in patients } \\
\text { with high } \\
\text { cardiovascular risk }\end{array}$ \\
\hline Total number of studies & $39(100 \%)$ & $4(100 \%)$ \\
\hline No. of patients in all studies & 4992 & 1230 \\
\hline Publication year, median (range) & 1996 (1974-2014) & 2013 (1992-2014) \\
\hline Publication year $<2000$ & $24(62 \%)$ & $1(25 \%)$ \\
\hline Multicentre studies & $9(23 \%)$ & $0(0 \%)$ \\
\hline Study size, median (IQR) & $84(54-129)$ & $251(210-406)$ \\
\hline Patient age, median (IQR) & $54(51-61)$ & $66(63-67)$ \\
\hline Male sex, median (IQR) & $62(25-87)$ & $77(66-88)$ \\
\hline \multicolumn{3}{|l|}{ Follow-up* } \\
\hline $0.5-1$ year & $7(18 \%)$ & $3(75 \%)$ \\
\hline$>1-3$ years & $21(54 \%)$ & $1(25 \%)$ \\
\hline$>3$ years & $11(28 \%)$ & $0(0 \%)$ \\
\hline \multicolumn{3}{|l|}{ Colchicine treatment (mg/day) } \\
\hline$\leq 1$ & $27(69 \%)$ & $3(75 \%)$ \\
\hline$>1$ & $12(31 \%)$ & $1(25 \%)$ \\
\hline \multicolumn{3}{|l|}{ Control treatment, $\mathrm{n}(\%)$} \\
\hline Active treatment & $8(21 \%)$ & $0(0 \%)$ \\
\hline Inactive, placebo & $31(79 \%)$ & $4(100 \%)$ \\
\hline \multicolumn{3}{|l|}{ Clinical setting } \\
\hline CVD, arteriosclerotic & $3(8 \%)$ & $3(75 \%)$ \\
\hline$C V D$, other & $1(3 \%)$ & $1(25 \%)$ \\
\hline Hepatobiliary disease & $25(64 \%)$ & $0(0 \%)$ \\
\hline Other & $10(26 \%)$ & $0(0 \%)$ \\
\hline \multicolumn{3}{|l|}{ Cardiovascular risk profile } \\
\hline Primary prevention & $0(0 \%)$ & $0(0 \%)$ \\
\hline Secondary prevention & $4(10 \%)$ & $4(100 \%)$ \\
\hline Not specified & $35(90 \%)$ & $0(0 \%)$ \\
\hline \multicolumn{3}{|l|}{ Risk of bias (high, unclear, low) } \\
\hline Randomisation & $3 \% / 59 \% / 38 \%$ & $0 \% / 25 \% / 75 \%$ \\
\hline Allocation concealment & $0 \% / 72 \% / 28 \%$ & $0 \% / 75 \% / 25 \%$ \\
\hline $\begin{array}{l}\text { Blinding of patients and } \\
\text { personnel }\end{array}$ & $18 \% / 13 \% / 69 \%$ & $25 \% / 0 \% / 75 \%$ \\
\hline Blinding of outcome assessment & $0 \% / 79 \% / 21 \%$ & $0 \% / 25 \% / 75 \%$ \\
\hline Incomplete outcome data & $41 \% / 38 \% / 21 \%$ & $0 \% / 50 \% / 50 \%$ \\
\hline Selective reporting & $13 \% / 84 \% / 3 \%$ & $0 \% / 100 \% / 0 \%$ \\
\hline
\end{tabular}

deemed the risk of bias lower across the four cardiovascular trials.

Funnel plots indicated no reporting bias for all-cause mortality, but adverse events seem to be selectively reported (online supplementary files 4-6).

\section{Quality of evidence}

The quality of evidence (GRADE) was deemed moderate in most cases due to imprecision. For adverse effects the quality was low or very low (table 2).

\section{Effects on patient-relevant outcomes}

All-cause mortality was reported in 30 trials $(\mathrm{n}=4174)$ with an RR of colchicine versus control of 0.94 (95\% CI 0.82 to 1.09 ; figure 2, online supplementary file 7). $.^{8-10} \quad 20-30$ w31-w45 w69 Between-study heterogeneity was low $\left(\mathrm{I}^{2}=27 \%\right)$. When we analysed only the four studies including patients with increased risk of cardiovascular events $(n=1230),{ }^{8-10} 20$ the RR was 0.54 (95\% CI 0.26 to 1.14; online supplementary file 7). This was stable in sensitivity analyses using alternative meta-analytical models (due to event rates close to $1 \%$; details in ${ }^{11}$ ). There was no heterogeneity $\left(\mathrm{I}^{2}=0 \%\right)$.

Myocardial infarction was reported in six studies $(\mathrm{n}=910){ }^{9} 192327$ w31 w69 One trial involving patients with high cardiovascular risk reported the number of total, fatal and non-fatal myocardial infarctions. ${ }^{9}$ Four trials reported only fatal myocardial infarctions, ${ }^{23} 27 \mathrm{w} 31$ w69 and in one trial no cardiovascular events occurred. ${ }^{19}$ We found a statistically significant reduction of total myocardial infarctions (RR $0.20 ; 95 \% \mathrm{CI}$ 0.07 to 0.57 ; figure 2). Since almost all reported events were non-fatal, results for non-fatal myocardial infarctions were similar (figure 2, table 3). Across all studies, only five fatal myocardial infarctions were reported and no significant effect was found when fatal myocardial infarction was analysed separately (OR 0.28 ; $95 \%$ CI 0.05 to 1.62 ; online supplementary file 8 ). There was little heterogeneity in all analyses $\left(\mathrm{I}^{2} \leq 13 \%\right)$.

Adverse events were reported in 13 trials; 11 reported any adverse events $(n=1313)^{27}$ w40 w41 w46-w53 and 11 reported gastrointestinal side effects specifically $(\mathrm{n}=1258) .^{10} 192027 \mathrm{w} 40$ w46 w47 w49 w51-w53 In four trials, the number of serious adverse events (SAEs) per study group was reported $(\mathrm{n}=472),{ }^{\mathrm{w} 47-\mathrm{w} 49}$ w53 and there were no events over 824 patient-years of follow-up. The RR of colchicine versus control on any adverse events was $1.52\left(95 \%\right.$ CI 0.93 to $2.46 ; \mathrm{I}^{2}=45 \%$; figure 2, online supplementary file 9). Colchicine was associated with more gastrointestinal side effects (RR 1.83; 95\% CI 1.03 to 3.26; $\mathrm{I}^{2}=74 \%$; figure 2 , online supplementary file 10 ). The relatively high heterogeneity was substantially reduced (to 19\%) when one trial with high rates of gastrointestinal events in both groups was excluded. ${ }^{19}$ Within the eight trials reporting total and gastrointestinal side effects, 66 of the 73 (90\%) reported events were for gastrointestinal reasons. For patients with increased cardiovascular risk, only data on gastrointestinal side effects from two studies were available but findings were consistent (RR 2.41; 95\% CI 1.43 to 4.06; table 3).

Cardiovascular mortality was reported in seven trials ( $\mathrm{n}=1132){ }^{9} 10192327$ w31 w69 The RR was 0.34 (95\% CI 0.09 to $1.21 ; \mathrm{I}^{2}=9 \%$; figure 2 , online supplementary file 11). Similar effects were found in sensitivity analyses with alternate meta-analytical models (Peto's OR 0.24; $95 \%$ CI 0.09 to 0.64 ; Mantel-Haenszel fixed-effects RR $0.20 ; 95 \%$ CI 0.06 to 0.68 ) and when we combined the two studies reporting this outcome in high cardiovascular risk populations (RR $0.25 ; 95 \%$ CI 0.02 to 2.66 ; table 3 ).

Few studies reported only few events on stroke, heart failure, non-scheduled hospitalisations and unscheduled cardiovascular interventions (figure 2, table 3). Colchicine significantly reduced non-scheduled hospitalisations for any reason (RR 0.87; 95\% CI 0.77 to $\left.0.99 ; \mathrm{I}^{2}=0 \%\right)$. Data specifically related to nonscheduled hospitalisations for cardiovascular reasons were not available. For other outcomes we found no statistically significant effects.

\section{Impact of colchicine dose}

We detected a statistically significant dose effect of colchicine on all-cause mortality in favour of lower dose treatment $(\mathrm{p}=0.03$ for interaction; online supplementary file 12). In studies using $0.5 \mathrm{mg} /$ day to $1 \mathrm{mg} /$ day colchicine $(\mathrm{n}=21 ; 2420$ patients), all-cause mortality was significantly reduced (RR $0.82 ; 95 \% \mathrm{CI}$ 0.67 to 0.99 ) in contrast to studies using higher doses (ie, up to $2 \mathrm{mg} /$ day; $\mathrm{n}=9 ; 1754$ patients) (RR 1.08; 95\% CI 0.93 to 1.25). The effect on adverse events was not modified (details $\left.\mathrm{in}^{11}\right)$. There was insufficient data for other outcomes. 
Table 2 Summary of findings table

\begin{tabular}{|c|c|c|c|c|c|}
\hline \multirow[b]{2}{*}{ Outcomes } & \multirow[b]{2}{*}{$\begin{array}{l}\text { No of participants } \\
\text { (studies) follow-up }\end{array}$} & \multirow[b]{2}{*}{$\begin{array}{l}\text { Quality of the evidence } \\
\text { (GRADE) }\end{array}$} & \multirow[b]{2}{*}{$\begin{array}{l}\text { Relative effect } \\
(95 \% \mathrm{CI})\end{array}$} & \multicolumn{2}{|c|}{ Anticipated absolute effects } \\
\hline & & & & $\begin{array}{l}\text { Risk with any control } \\
\text { treatment }\end{array}$ & $\begin{array}{l}\text { Risk difference with colchicine } \\
(95 \% \mathrm{Cl})\end{array}$ \\
\hline Mortality (all-cause) & $\begin{array}{l}4174 \text { (30 studies) } \\
0.5-14 \text { years }\end{array}$ & $\begin{array}{l}\oplus \oplus \oplus \Theta \text { MODERATE * } \\
\text { due to imprecision }\end{array}$ & RR 0.94 (0.82 to 1.09 ) & 193 per 1000 & $\begin{array}{l}12 \text { fewer per } 1000 \text { (from } 37 \\
\text { fewer to } 17 \text { more) }\end{array}$ \\
\hline $\begin{array}{l}\text { Subgroup: Patients with } \\
\text { high cardiovascular risk }\end{array}$ & $\begin{array}{l}1230 \text { ( } 4 \text { studies) } \\
0.5-3 \text { years }\end{array}$ & $\begin{array}{l}\oplus \oplus \oplus \Theta \text { MODERATE * } \\
\text { due to imprecision }\end{array}$ & RR 0.54 (0.26 to 1.14$)$ & 32 per 1000 & $\begin{array}{l}15 \text { fewer per } 1000 \text { (from } 24 \\
\text { fewer to } 4 \text { more) }\end{array}$ \\
\hline $\begin{array}{l}\text { Mortality } \\
\text { (cardiovascular) }\end{array}$ & $\begin{array}{l}1132 \text { (7 studies) } \\
0.5-14 \text { years }\end{array}$ & $\begin{array}{l}\oplus \oplus \oplus \Theta \text { MODERATE * } \\
\text { due to imprecision }\end{array}$ & RR 0.34 (0.09 to 1.21 ) & 27 per 1000 & $\begin{array}{l}18 \text { fewer per } 1000 \text { (from } 24 \\
\text { fewer to } 6 \text { more) }\end{array}$ \\
\hline $\begin{array}{l}\text { Subgroup: Patients with } \\
\text { high cardiovascular risk }\end{array}$ & $\begin{array}{l}754 \text { ( } 2 \text { studies) } \\
0.5-3 \text { years }\end{array}$ & $\begin{array}{l}\oplus \oplus \Theta \Theta \text { LOW } † \ddagger \text { due to } \\
\text { imprecision }\end{array}$ & RR 0.25 (0.02 to 2.66$)$ & 31 per 1000 & $\begin{array}{l}31 \text { fewer per } 1000 \text { (from } 30 \\
\text { fewer to } 51 \text { more) }\end{array}$ \\
\hline $\begin{array}{l}\text { Myocardial Infarction } \\
\text { (total)§ }\end{array}$ & $\begin{array}{l}652 \text { ( } 2 \text { studies } 1) \\
3 \text { years }\end{array}$ & $\begin{array}{l}\oplus \oplus \oplus \ominus \text { MODERATE ** } \\
\text { due to imprecision }\end{array}$ & RR 0.20 (0.07 to 0.57$)$ & 58 per 1000 & $\begin{array}{l}46 \text { fewer per } 1000 \text { (from } 25 \\
\text { fewer to } 54 \text { fewer) }\end{array}$ \\
\hline $\begin{array}{l}\text { Subgroup: Patients with } \\
\text { high cardiovascular risk§ }\end{array}$ & $\begin{array}{l}532 \text { ( } 1 \text { study) } \\
3 \text { years }\end{array}$ & $\begin{array}{l}\oplus \oplus \oplus \ominus \text { MODERATE ** } \\
\text { due to imprecision }\end{array}$ & RR 0.20 (0.07 to 0.57$)$ & $\begin{array}{l}\text { Study population } \\
72 \text { per } 1000 \\
\text { Extrapolated 1-year ris } \\
25 \text { per } 1000\end{array}$ & $\begin{array}{l}58 \text { fewer per } 1000 \text { (from } 31 \\
\text { fewer to } 67 \text { fewer) } \\
20 \text { fewer per } 1000 \text { (from } 11 \\
\text { fewer to } 23 \text { fewer) }\end{array}$ \\
\hline 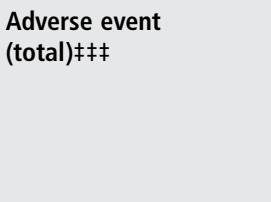 & $\begin{array}{l}1313 \text { (11 studies) } \\
0.5-14 \text { years }\end{array}$ & $\begin{array}{l}\oplus \Theta \Theta \Theta \text { VERY LOW }{ }^{*}, \ddagger \ddagger, \S \S \\
\text { due to risk of bias, } \\
\text { imprecision, publication bias }\end{array}$ & RR 1.52 (0.93 to 2.46$)$ & $\begin{array}{l}\text { Study population } \\
89 \text { per } 1000 \\
\text { Assumed } 1 \text {-year risk } \\
89 \text { per } 1000\end{array}$ & $\begin{array}{l}46 \text { more per } 1000 \text { (from } 6 \\
\text { fewer to } 130 \text { more) } \\
46 \text { more per } 1000 \text { (from } 6 \\
\text { fewer to } 130 \text { more) }\end{array}$ \\
\hline $\begin{array}{l}\text { Adverse event } \\
\text { (gastrointestinal) }\end{array}$ & $\begin{array}{l}1258 \text { (11 studies) } \\
0.5-14 \text { years }\end{array}$ & $\begin{array}{l}\oplus \oplus \Theta \Theta \text { LOW } \S \S \text {, १ी due to } \\
\text { inconsistency, publication } \\
\text { bias }\end{array}$ & RR 1.83 (1.03 to 3.26$)$ & $\begin{array}{l}\text { Study population } \\
132 \text { per } 1000 \\
\text { Assumed 1-year risk } \\
132 \text { per } 1000\end{array}$ & $\begin{array}{l}110 \text { more per } 1000 \text { (from } 4 \\
\text { more to } 299 \text { more) } \\
110 \text { more per } 1000 \text { (from } 4 \\
\text { more to } 298 \text { more) }\end{array}$ \\
\hline Adverse event (serious) & $\begin{array}{l}472 \text { ( } 4 \text { studies) } 824 \\
\text { patient-years }\end{array}$ & $\begin{array}{l}\oplus \oplus \Theta \Theta \text { LOW }^{* * *}, \text { t†† due } \\
\text { to imprecision, publication } \\
\text { bias }\end{array}$ & Not estimable & $\begin{array}{l}\text { No illustration of compar } \\
\text { assumed risks. }\end{array}$ & ative risks due to very uncertain \\
\hline Heart failure (total) & $\begin{array}{l}426 \text { ( } 3 \text { studies) } \\
0.5-3 \text { years) }\end{array}$ & $\begin{array}{l}\oplus \oplus \Theta \Theta \text { LOW } \neq, \mathbf{q}_{1}^{* *} \text { due to } \\
\text { imprecision, publication bias }\end{array}$ & RR 0.62 (0.1 to 3.88 ) & $\begin{array}{l}\text { No illustration of compar } \\
\text { assumed risks. }\end{array}$ & ative risks due to very uncertain \\
\hline Stroke (total) & $\begin{array}{l}874 \text { ( } 3 \text { studies) } \\
0.5-3 \text { years }\end{array}$ & $\begin{array}{l}\oplus \oplus \Theta \Theta \text { LOW } \neq, \boldsymbol{\eta}^{* *} \text { due to } \\
\text { imprecision, publication bias }\end{array}$ & OR 0.38 (0.09 to 1.7 ) & $\begin{array}{l}\text { No illustration of compar } \\
\text { assumed risks. }\end{array}$ & ative risks due to very uncertain \\
\hline \multicolumn{6}{|c|}{ 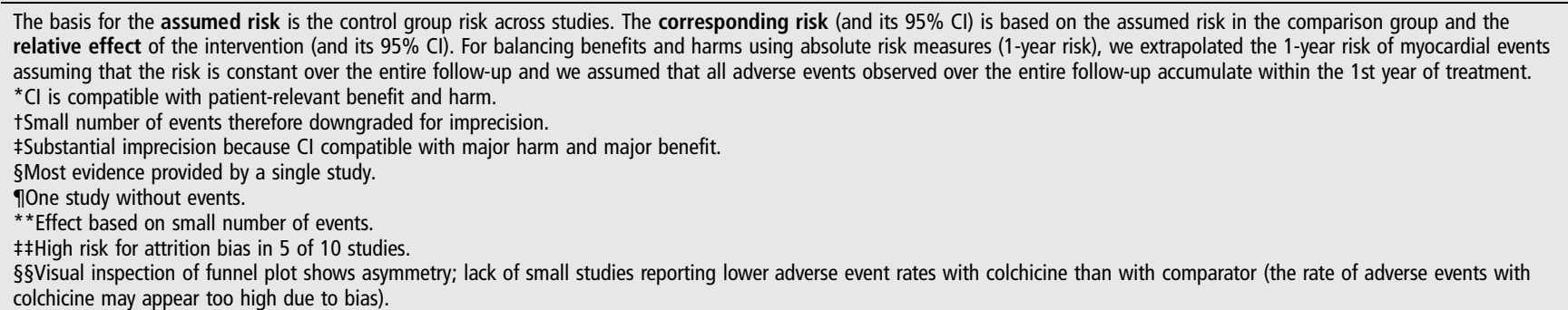 } \\
\hline
\end{tabular}

\section{Impact of control treatment and risk of bias}

We found no interaction between colchicine effects and the type of control treatment, potential selection bias, double-blinding, blinded outcome assessment, incomplete outcome data, or of whether studies were published as abstract or in full text $\left(\right.$ details in $\left.{ }^{11}\right)$.

\section{DISCUSSION}

We included 39 RCTs with follow-up to 14 years comparing colchicine with any control in 4992 patients with any condition or disease. Colchicine had no significant effect on all-cause mortality across all studies. Moderate quality evidence suggests an $80 \%$ RR reduction for myocardial infarction although most of the evidence was provided by a single study. Such large effects ranging between 0.2 and 0.34 (RR and OR, respectively) were also seen for cardiovascular mortality, which was significantly reduced in some but not all meta-analytical models. The observed 13\% RR reduction for non-scheduled hospitalisations is not clearly attributable to underlying cardiovascular effects. 


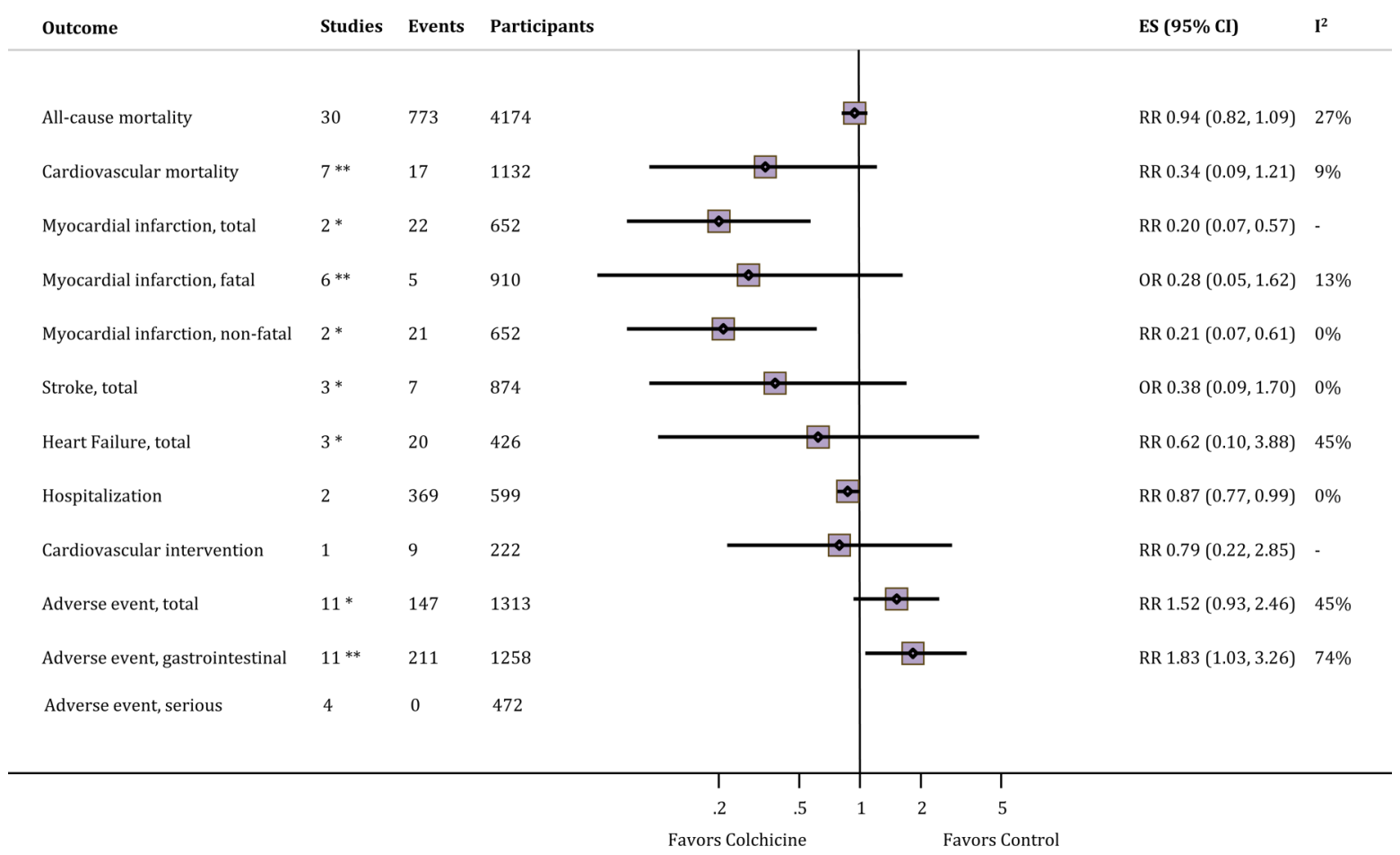

Figure 2 Overview of meta-analyses for colchicine treatment versus control on various patient-relevant outcomes.

ES, effect estimate; OR, Peto's OR; RR, relative risk. *Including one study without events. ${ }^{* *}$ Including two studies without events.

As expected, colchicine increased the risk of gastrointestinal side effects ( $83 \%$ increased RR). These were typically described as mild and transient, including diarrhoea, nausea, abdominal pain or vomiting. In contrast, we found no evidence indicating increased risks for SAEs over 824 patient-years, although the quality of evidence was low.

Results for stroke, heart failure and non-scheduled cardiovascular interventions were inconclusive due to wide CIs.
Balancing potential benefits and harms, we estimate that treating 1000 patients over 1 year with colchicine for secondary prevention of cardiovascular disease may protect 20 patients (1123) from experiencing a myocardial infarction at the cost of 110 (4-299 more) having mostly mild and transient gastrointestinal intolerance (table 2). The potential clinical impact of 3 years of treatment on prevention of myocardial infarction can be estimated by a number-needed-to-treat of 17 (assuming a

Table 3 Results for patients with high cardiovascular risk

\begin{tabular}{|c|c|c|c|c|c|c|}
\hline Outcome & Studies (n) & Events (n) & Patients (n) & Summary effect $(95 \% \mathrm{Cl})$ & Heterogeneity $\left(1^{2}\right), \%$ & Subgroup effect ( $p$ Value) \\
\hline \multicolumn{7}{|l|}{ Patients with high cardiovascular risk } \\
\hline All-cause mortality & 4 & 29 & 1230 & RR 0.54 (0.26 to 1.14$)$ & $0 \%$ & 0.13 \\
\hline Cardiovascular mortality & 2 & 13 & 754 & RR 0.25 (0.02 to 2.66$)$ & $49 \%$ & n.c. \\
\hline \multicolumn{7}{|l|}{ Myocardial infarction } \\
\hline Fatal or non-fatal & 1 & 22 & 532 & RR 0.20 (0.07 to 0.57 ) & - & n.c. \\
\hline Fatal & 1 & 1 & 532 & RR 0.30 (0.01 to 7.22 ) & - & n.c. \\
\hline Non-fatal & 1 & 21 & 532 & RR 0.21 (0.07 to 0.61$)$ & - & n.c. \\
\hline \multicolumn{7}{|l|}{ Stroke } \\
\hline Fatal or non-fatal & 2 & 7 & 754 & OR 0.38 (0.09 to 1.70$)$ & $0 \%$ & n.c. \\
\hline Fatal & 2 & 1 & 754 & OR 7.26 (0.14 to 365.85$)$ & - & n.c. \\
\hline Non-fatal & 2 & 6 & 754 & OR 0.23 (0.05 to 1.17$)$ & $0 \%$ & n.c. \\
\hline \multicolumn{7}{|l|}{ Heart failure } \\
\hline Fatal or non-fatal & 1 & 3 & 222 & RR 0.14 (0.01 to 2.69 ) & - & n.c. \\
\hline Fatal & 1 & 1 & 222 & RR 0.33 (0.01 to 7.95 ) & - & n.c. \\
\hline Non-fatal & 1 & 2 & 222 & RR 0.20 (0.01 to 4.05$)$ & - & n.c. \\
\hline Hospitalisation & 0 & - & - & - & - & - \\
\hline Cardiovascular intervention & 1 & 9 & 222 & RR 0.79 (0.22 to 2.85$)$ & - & n.c. \\
\hline Adverse event, any & 0 & - & - & - & - & - \\
\hline Adverse event, gastrointestinal & 2 & 62 & 501 & RR 2.41 (1.43 to 4.06 ) & $0 \%$ & n.c. \\
\hline
\end{tabular}


baseline risk of $72 / 1000$ as in the control group of that trial providing most of the evidence for myocardial infarction; ${ }^{9}$ table 2 ).

There was no evidence that a daily dose of $>1 \mathrm{mg}$ improves survival (in contrast, lower doses may be associated with mortality benefits). This should inform further research on the optimal treatment dose, in particular for many indications where the available trial evidence is insufficient to address dosing questions, including gout, pericarditis and FMF. ${ }^{7}$ w5 w55 However, although this analysis was specified a priori, we did not anticipate stronger effects with lower doses; it might be a chance finding, and this underlines that there is still much to be learned about the clinical use of colchicine.

Strengths of our study are that we followed established methods for systematic reviewing and evidence synthesis, explored various sources of potential bias, used different statistical approaches to scrutinise results, used an upfront published study protocol, applied a highly sensitive literature search and systematically contacted study authors to integrate unpublished data. Previous meta-analyses addressed specific conditions, including FMF, ${ }^{\text {w56 }}$ gout, ${ }^{\text {w54 }}$ pericarditis, ${ }^{\text {w55 }}$ w57-w59 atrial fibrillation, ${ }^{\text {w60 }}$ liver fibrosis and cirrhosis ${ }^{\mathrm{w} 61}$ or primary biliary cirrhosis $^{\text {w62 }}$ but evaluated safety only in relatively narrow spectrums of patients. We kept a broad perspective by applying wide eligibility criteria to include populations with any disease or clinical condition. This allowed us to evaluate colchicine effects, particularly safety, independently of the medical indication and using the entire clinical trial evidence. ${ }^{\text {w63 }}$ To our knowledge this is the first systematic review and meta-analysis on colchicine that evaluated cardiovascular outcomes in any patient population and the largest analysis of randomised evidence on colchicine safety. Our results are in keeping with the main findings of a recently published systematic review, which was limited to patients with cardiac disease, ${ }^{\text {w70 }}$ that indicates potential cardiovascular benefits of colchicine but also highlights the need for further randomised evidence. We considered various patient-relevant outcomes and by including adverse events as primary outcome we aimed to allow a balanced assessment of colchicine. ${ }^{\mathrm{w} 64}$

There are a number of important caveats that need to be considered. First, as for all systematic reviews, the validity of our results depends on the methodological quality of the included studies. We evaluated RCTs which were mostly placebocontrolled, double-blinded and some explicitly mentioned blinded outcome assessment (most of the observed fatal cardiovascular events and myocardial infarctions were assessed by blinded outcome assessors). Bias due to missing outcome data (because substantial proportions of randomised patients were not followed up or completeness was unbalanced between study groups) could not be ruled out for many studies but this was less of a problem for the cardiovascular trials, especially the trial contributing most evidence to cardiovascular effects ${ }^{9}$ had a low risk of bias in this regard. In sensitivity analyses, we found no indication that such bias affected the results.

Second, the results depend on the reporting quality of the primary studies and reporting of adverse events is known to be problematic. ${ }^{\text {w65 }}$ Most trials were published long before reporting guidelines were established and some studies were published as abstracts only. Cardiovascular outcomes were often only casually reported as adverse events, typically among the causes of death. Some studies selectively described SAEs that the investigators deemed related to the study drug. Some studies reported that no SAEs occurred while there were events which would meet the definition of an SAE (such as deaths, myocardial infarctions or strokes). The funnel plots for adverse events showed asymmetry suggesting reporting bias and thus the increased risk of adverse events might be overestimated. In addition, reporting of adverse events was frequently limited to the most common ones. However, cardiovascular events were uncommon in most eligible trials which were not designed to evaluate such effects. Thus, reporting of non-fatal events, cardiovascular or not, was probably often neglected. Only studies focusing specifically on cardiovascular topics systematically reported some cardiovascular outcomes. Yet, despite wide CIs, the effect estimates for studies on non-cardiovascular topics were similar to those for studies systematically assessing cardiovascular effects and we also found no substantial between-study heterogeneity (although heterogeneity tests are limited due to low number of studies). ${ }^{17}$ Moreover, the study that contributed most of the evidence on myocardial infarction ${ }^{9}$ clearly reported the ascertainment of such events. We aimed to address the reporting problem and could, for at least some trials, obtain complete information on cardiovascular outcomes by contacting study authors.

Third, there were few events in some meta-analyses and some outcome results were dominated by a single study, which is a clear limitation. For example effects on myocardial infarction are based on only a small proportion of all randomised patients. However, effect sizes showed a consistent pattern across various scenarios with respect to non-fatal myocardial infarction (RR 0.21 ; results are mainly driven by a large study specifically designed for cardiovascular effects), fatal myocardial infarction (OR 0.28; some small studies without systematic outcome assessment), cardiovascular mortality (RR 0.34; seven studies in diverse clinical settings) and all-cause mortality (RR 0.54; four studies with patients of high cardiovascular risk).

Fourth, we did not assess effects on inflammatory markers or surrogate outcomes because we focused on outcomes most important for patients and clinical decision making. Among the studies in patients with cardiovascular disease, one trial reported changes of levels of high-sensitivity CRP (C-reactive protein) interleukin 6 and demonstrated significant reductions for both biomarkers. $^{20}$

Fifth, there was insufficient information on concomitant cardiovascular medication, for example, aspirin, statins. However, in the study contributing most information on effects in patients with established cardiovascular disease, ${ }^{9}$ almost all patients used statins and antiplatelet therapy. This suggests that colchicine effects are additional to concomitant standard treatments.

Finally, we found a dose effect when we dichotomised studies according to allowed dosage. Some studies used individualised dose regimens within a certain range and we had no information on the actually used median dose across all patients in such studies. This reduced the granularity of the data and thus we did not perform metaregression to further analyse the dose effect. Individual patient data meta-analyses are needed to further elucidate the question on the optimal treatment dose.

Our comprehensive analysis suggests that colchicine is a promising novel treatment option for cardiovascular disease-a medical field where breakthrough preventive treatments are eagerly awaited for years. Although moderate quality evidence indicates cardiovascular benefits, especially on myocardial infarction, there is sufficient uncertainty surrounding the benefits and harms of colchicine to interpret this cautiously until more randomised trial evidence is available.

In trial registries, we identified three ongoing RCTs in cardiovascular populations (COLPET, ${ }^{\text {w66 }}$ COACS $^{\text {w67 }}$ and LoDoCo $\left.2^{\mathrm{w} 68}\right)$. In a double-blinded design, they compare effects of low dose colchicine $(0.5 \mathrm{mg} / \mathrm{day}$ and $0.6 \mathrm{mg} / \mathrm{day})$ versus 
placebo on cardiovascular outcomes. They aim to follow-up about 100 patients over 6 months, 500 over 2 years and $>2000$ over 3-5 years, planned to be completed in December 2015, ${ }^{\text {w66 }}$ June $2016,{ }^{\text {w67 }}$ and 2018 or $2019,{ }^{\text {w68 }}$ respectively.

The potentially large benefits of this inexpensive treatment on patient-important clinical outcomes and mortality should encourage funding and conduct of large-scale randomised trials to further explore the merits of colchicine in cardiovascular disease.

Acknowledgements The authors thank Mark A Berendsen and Nicole Martin for conducting the literature searches, Kübra Özoglu for her excellent administrative support and assistance with literature management, Tiago V Pereira, Benjamin Kasenda, Cemile Bathelt-Cicek for assessing studies reported in Portuguese, Chinese and Turkish. The authors thank the Cochrane Heart Group for their support in publishing the Cochrane Review. The authors thank all study investigators for providing helpful information on their studies.

Contributors LGH and MB conceived the study. LGH, HE, VLG, AA, KKO, DG, AJN and MB extracted the data. LGH and HE analysed the data. LGH, HE, AJN, MN and MB interpreted the results. LGH and HE wrote the first draft and all authors critically revised the manuscript. All authors read and approved the final version of the paper. LGH is guarantor.

Competing interests All authors declare no financial relationships with any organization that might have an interest in the submitted work in the previous 3 years. Mark Nidorf was involved in one of the included studies, which was investigator initiated and conducted without external financial support. All other authors declare no other relationships or activities that could appear to have influenced the submitted work.

Provenance and peer review Not commissioned; externally peer reviewed.

\section{REFERENCES}

1 Ridker PM, Luscher TF. Anti-inflammatory therapies for cardiovascular disease. Eur Heart J 2014;35:1782-91.

2 Libby P. Mechanisms of acute coronary syndromes and their implications for therapy. N Engl J Med 2013;368:2004-13.

3 Shah PK. Inflammation and plaque vulnerability. Cardiovasc Drugs Ther 2009:23:31-40.

4 Niel E, Scherrmann JM. Colchicine today. Joint Bone Spine 2006;73:672-8.

5 Terkeltaub RA. Colchicine update: 2008. Semin Arthritis Rheum 2009;38:411-19.

6 Cocco G, Chu DC, Pandolfi S. Colchicine in clinical medicine. A guide for internists. Eur J Intern Mede 2010:21:503-8.

7 Hentgen V, Grateau G, Kone-Paut I, et al. Evidence-based recommendations for the practical management of Familial Mediterranean Fever. Semin Arthritis Rheum 2013:43:387-91

8 O'Keefe JH Jr, McCallister BD, Bateman TM, et al. Ineffectiveness of colchicine for the prevention of restenosis after coronary angioplasty. J Am Coll Cardiol 1992:19:1597-600

9 Nidorf SM, Eikelboom JW, Budgeon CA, et al. Low-dose colchicine for secondary prevention of cardiovascular disease. J Am Coll Cardiol 2013;61:404-10.
10 Deftereos S, Giannopoulos G, Raisakis K, et al. Colchicine treatment for the prevention of bare-metal stent restenosis in diabetic patients. J Am Coll Cardiol 2013;61:1679-85.

11 Hemkens LG, Ewald H, Gloy VL, et al. Colchicine for prevention of cardiovascular events. Cochrane Database of Systematic Reviews 2016, Issue 1. Art. No.: CD011047. doi:10.1002/14651858.CD011047.pub2

12 Hemkens Lars G, Gloy Viktoria L, Olu Kelechi K, et al. Colchicine for prevention of cardiovascular events. Cochrane Database Syst Rev 2014;(3). http://onlinelibrary. wiley.com/doi/10.1002/14651858.CD011047/abstract

13 Hemkens L, Gloy V, Olu K, et al. Colchicine for prevention of cardiovascular events [Cochrane Protocol]. PROSPERO 2015:CRD42015017733. Secondary Colchicine for prevention of cardiovascular events[Cochrane Protocol]. PROSPERO 2015: CRD42015017733 2015. http://www.crd.york.ac.uk/PROSPERO/display_record.asp? ID=CRD42015017733

14 Higgins JPT, Green S, eds. Cochrane handbook for systematic reviews of interventions[Internet]. Version 5.1.0[updated March 2011]. The Cochrane Collaboration, 2011 [cited 08 Jan 2013].

15 Guyatt GH, Oxman AD, Vist GE, et al. GRADE: an emerging consensus on rating quality of evidence and strength of recommendations. BMJ 2008:336:924-6.

16 Lau J, Ioannidis JP, Schmid CH. Quantitative synthesis in systematic reviews. Ann Intern Med 1997:127:820-6.

17 Ioannidis JP, Patsopoulos NA, Evangelou E. Uncertainty in heterogeneity estimates in meta-analyses. BMJ 2007;335:914-16.

18 Bradburn MJ, Deeks JJ, Berlin JA, et al. Much ado about nothing: a comparison of the performance of meta-analytical methods with rare events. Stat Med 2007:26:53-77.

19 Yurdakul S, Mat C, Tuzun Y, et al. A double-blind trial of colchicine in Behcet's syndrome. Arthritis Rheum 2001;44:2686-92.

20 Deftereos S, Giannopoulos G, Panagopoulou V, et al. Anti-inflammatory treatment with colchicine in stable chronic heart failure: a prospective, randomized study. JACC Heart Failure 2014;2:131-7.

21 Kershenobich D, Uribe M, Suarez G, et al. Treatment of cirrhosis with colchicine: a randomized trial[abstract]. Gastroenterology 1976;70:A128/986.

22 Kyle RA, Greipp PR, Garton JP, et al. Primary systemic amyloidosis. Comparison of melphalan/prednisone versus colchicine. Am J Med 1985;79:708-16.

23 Kaplan MM, Alling DW, Zimmerman $\mathrm{HJ}$, et al. A prospective trial of colchicine for primary biliary cirrhosis. N Engl J Med 1986;315:1448-54.

24 Reinhardt M, Jorke D, Jahn G, et al. Colchicine therapy of fibrosing liver diseasesreport of a randomized double-blind study. Deutsche Zeitschrift fur Verdauungsund Stoffwechselkrankheiten 1986;46:257-75.

25 Warnes TW, Smith A, Lee Fl, et al. A controlled trial of colchicine in primary biliary cirrhosis. Trial design and preliminary report. J Hepatol 1987;5:1-7.

26 Bodenheimer $\mathrm{H} \mathrm{Jr}$, Schaffner F, Pezzullo J. Evaluation of colchicine therapy in primary biliary cirrhosis. Gastroenterology 1988:95:124-9.

27 Kershenobich D, Vargas F, Garcia-Tsao G, et al. Colchicine in the treatment of cirrhosis of the liver. N Engl J Med 1988;318:1709-13.

28 Trinchet JC, Beaugrand M, Callard P, et al. Treatment of alcoholic hepatitis with colchicine. Results of a randomized double blind trial. Gastroenterol Clin Biol 1989;13:551-5

29 Buligescu L, Voiculescu M. Randomized controlled clinical trial of colchicine in the long term treatment of liver cirrhosis[EASL abstract]. J Hepatol 1989;9(Suppl 1):S12.

30 Wang YJ, Lee SD, Hsieh MC, et al. A double-blind randomized controlled trial of colchicine in patients with hepatitis B virus-related postnecrotic cirrhosis. J Hepatol 1994;21:872-7. 